\title{
Adaptive Multi-Objective Optimization Scheme for Cognitive Radio Resource Management
}

\author{
Ismail AlQerm and Basem Shihada \\ CEMSE Division, KAUST, Saudi Arabia, \{ismail.qerm, basem.shihada\}@kaust.edu.sa
}

\begin{abstract}
Cognitive Radio (CR) is an intelligent Software Defined Radio (SDR) that is capable to alter its transmission parameters according to predefined objectives and wireless environment condition. Cognitive engine exploits optimization and machine learning techniques to perform radio parameters configuration. In this paper, we propose an Adaptive Multiobjective Optimization Scheme (AMOS) for cognitive radio resource management to improve spectrum operation and network performance. The optimization relies on adapting radio transmission parameters to environment conditions using constrained optimization modeling called fitness functions in an iterative manner. These functions include minimizing power consumption, packet error rate (PER), delay and interference. On the other hand, maximizing throughput and spectral efficiency. Cross-layer optimization is exploited to access environmental parameters from all TCP/IP stack layers. AMOS uses Genetic Algorithm (GA) that is adaptive in terms of its parameters and objective weights as the vehicle of optimization. The proposed scheme has demonstrated quick response and efficiency in three different scenarios compared to other schemes. In addition, it demonstrate its capability to optimize the performance of other TCP/IP layers in addition to the physical layer.

Index Terms-Cross-layer optimization, transmission parameters, decision-making, genetic algorithm; cognitive radio
\end{abstract}

\section{INTRODUCTION}

The demand for wireless and mobile communications has been rapidly growing, which is expected to cause the spectrum-scarcity problem soon. This trend motivates researchers to find new optimization and enabling technologies to improve the efficiency of wireless communications and cope with the current spectrum demands. Cognitive radio (CR), first introduced by Mitola in 1999 [1], is a promising technology to improve spectrum utilization and network efficiency. It is often realized with Software Defined Radio (SDR) [2] augmented with machine learning and optimization algorithms that are capable to adapt radio transmission parameters to communication environment conditions. Mitola's vision was to build a cognitive engine for resource management that is capable to sense, observe, decide and act to improve network performance. However, most of the approaches proposed thus far [3][4] consider dynamic spectrum access (DSA) as the main goal of CR, which was not part of Mitola's vision. Few architectures considered using optimization in resource management but none of them comprehensively exploits TCP/IP layers for radio adaptation. Application QoS metrics such as throughput, delay, Bit Error Rate (BER), etc. are key factors of radio resource management in the design of any cognitive optimization scheme. Therefore, We call this constrained optimization as it is determined by application-layer requirements, and channel conditions. Environmental parameters from all TCP/IP stack layers (not just physical layer) should be considered for more comprehensive decisions as it reflects the surrounding environment. Cross-layer optimization is an ultimate approach to extract environmental parameters and QoS requirements. Environment awareness information is then sent to the cognitive engine to reason about and decide on the best possible configuration for the transmission parameters of the incumbent channel. Details of cross-layer optimization can be found in [5].

There are many optimization approaches for radio resource management and transmission parameters configuration in the cognitive context, such as rule-based systems [6], fuzzy logic [7], and neural networks [8]. These approaches can find solutions but may not produce best ones as they have various limitations in adapting parameters. For example, rulebased systems are limited to predetermined capabilities by their own rule set. Fuzzy logic approximated solutions are not based on certain input, and hence, it does not provide accurate solutions. Neural networks include extensive training to generate observed behavior, but become unstable when constraints are necessary to accounted for. An Ant Colony Optimization (ACO) algorithm is used to solve the optimization problem by formulating it into single objective function [9]. Particle Swarm Optimization (PSO) is also being used in [10] to determine the CR transmission parameters for a given set of objectives. Game theory is in a nascent stage and used for interactive decision-making, but provides analytical tools to predict the outcome of complex interactions among the rational entities based on perceived results. However, the dynamic nature of real networks makes the implementation of game theory in cognitive radios very complex. In addition, the heterogeneity of cognitive radio networks and the multiple layers involved in radio configuration exacerbate difficulties and drawbacks in the above optimization techniques. In [11], Simulated Annealing (SA) cross-layer optimization is exploited to improve communication quality based on utility function principle. Transmission parameters are tuned based on the utility functions calculation for certain communication quality metrics such as throughput, delay...etc. The aim of this optimization is to maximize these utility values. However, the transmission parameters considered in this optimization are limited to specific scenarios and not covering all possible optimization problems. Although SA is fast in convergence, it does not improve with time which is necessary as more search is required to find solutions in such dynamic environment with 
plenty of parameters and multiple objectives.

In this paper, we propose an Adaptive Multi-Objective Optimization scheme (AMOS) that is capable to handle multiple competing objectives with consideration of policy limitations. It exploits cross-layer design for radio environment awareness and Genetic Algorithm (GA) as an optimization tool [4] for selection of transmission parameters. GA is well suited for multi-objectives optimization problems in cognitive radio networks as it can search for multiple sets of solutions over a large search space, and can enforce constraints [12]. Fast convergence, ease of implementation as well as optimization of discrete and continuous radio parameters render GA as an excellent optimization tool for making resource management decisions in cognitive radio networks [13]. AMOS aims to meet QoS requirements which include throughput, delay, and BER, and improves spectrum efficiency. It is composed of six fitness functions that support minimization of power consumption, reduction of BER, minimization of delay and interference, and maximization of throughput and spectral efficiency. To ensure that GA runs effectively and exploit all solutions possibilities, AMOS adjusts GA parameters (crossover and mutation) dynamically to fit with the available search space and this helps GA not be stuck in local optima. In addition, the following salient features distinguished AMOS from other cognitive decision-making schemes.

1) All TCP/IP stack layers in the radio environment are considered for environmental parameters input. This improves accuracy and adaptivity, and enables efficient transmission.

2) The objective functions used in AMOS are comprehensive by incorporating throughput, delay, PER, spectral efficiency, power, and interference.

3) AMOS is not limited to point-to-point link optimization and accounts for the environment where cognitive links are non-cooperative.

4) The weights and priorities of objective functions are adapted automatically according to the environment conditions and system capability.

The rest of the paper is organized as follows. Section II presents the concept of constrained multi-objective optimization in cognitive radio. Our constrained optimization functions for AMOS are also presented there. Section III describes the AMOS mechanism. Section IV evaluates the performance of AMOS in terms of throughput and compares its convergence with other schemes. The paper concludes with Section V.

\section{Constrained Multi-Objective Optimization in Cognitive Radios}

Assuming a multicarrier dynamic wireless environment with multiple subcarriers, the basic characteristic of $\mathrm{CR}$ is to sense the environmental parameters, and it runs optimization to adjust the value of transmission parameters to achieve the predefined quality of service (QoS). In this section, we define the cognitive optimization problem and the associated parameters in the optimization process. In addition, our multi- objective optimization model is described with all related fitness functions.

\section{A. Multi-Objective Optimization Problem}

A multi-objective optimization problem is to determine the optimal value of a set of solutions $(x)$ while optimizing a set of $k$ conflicting objectives simultaneously. Mathematically, the multi-objective optimization problem is defined as

$$
\min / \max (Y)=f(x)=\left[f_{1}(x), f_{2}(x), \ldots, f_{n}(x)\right]
$$

subject to $x=\left(x_{1}, x_{2}, \ldots, x_{m}\right) \in X$ and $y=$ $\left(y_{1}, y_{2}, \ldots, y_{n}\right) \in Y$ where there are $n$ dimensional spaces, $f(x)$ is the fitness function for a certain dimension. $X$ is the set of transmission parameters and $Y$ the set of dimensions. The fitness function is a mathematical equation that is evaluated to reach certain optima according the radio environmental parameters and QoS requirements. They are normalized to have score between 0 and 1 . The CR parameters adaptation problem can be modeled as a multi-objective optimization problem with the goal to find $X$ that minimize or maximize the fitness function. The objectives under consideration might conflict with each other. For example, minimizing power and minimizing BER simultaneously creates a conflict due to the single parameter, transmit power, affecting each objective in a different way. The optimal set for multiple objective functions lie on what is known as the Pareto optimal front. This front represents the set of solutions that cannot be improved upon in any dimension. The solutions on the Pareto front are optimal and co-exist due to the trade-offs between the multiple objectives. These trade-offs represent the core of the multiple objective optimization problem. Therefore, CR engine must performs actions based on a single set of parameters, which should be selected from the Pareto front.

\section{B. Radio Parameters}

Radio parameters of CR are categorized into environmental parameters and transmission parameters. The former gives knowledge of environmental characteristics of the wireless environment and used as inputs to the $\mathrm{CR}$ engine. Environmental parameters include path loss $(P L)$, noise power $(N)$, signalto-noise ratio $(S N R)$, Acks of successfully received packets, spectrum occupancy information, fading statistics, Frame Error Rate $(F E R)$ and Bit Error rate $(B E R)$. Transmission parameters are the output parameters of the CR engine. This engine adjusts the transmission knobs to corresponding values from the optimal parameter set. The transmission parameters are listed as: transmission power $(P)$, type of modulation scheme used for the communication, modulation index $(m)$, bandwidth $(B)$, channel coding rate $R_{c}$, time division duplex $(T D D)$ in percentage, and symbol rate $\left(R_{s}\right)$ for the physical layer, frame size $(L)$, contention window size $(C W)$, and source coding for the MAC layer, transmission range $(d)$, and number of hops for the network layer, and congestion control mechanism for the transport layer. Modulation Index is defined as the total number of symbols in a modulation scheme and TDD represent the percentage of transmit time. 


\section{Objective Fitness Functions}

In our scheme (AMOS), we adopt the weighted sum approach to find the unified optimization function because it captures the trade-offs between multiple objectives. The weighted sum approach method suits the cognitive radio environment well since it provides a convenient process for applying weights to the objectives. Changing the objective direction of the fitness function requires only a simple change of the weighting factor. AMOS adjusts the weighing factors automatically according to application and environment awareness information. This information determine which of the objective functions is contributing more to meet the application QoS requirements. For example, a radio in default mode may be operating so to ensure the best throughput possible while not caring much about minimizing power. However, assuming this is a battery powered radio, the system may sense low power in the battery and modify the objective weights to emphasize minimizing power. In addition, the derived individual objective fitness functions are bounded by threshold values based on the applications QoS requirements. This will narrow the search space and help to enforce policy regulation by specifying certain range for each transmission parameter.

AMOS optimization consists of the following fitness functions: power, BER, interference, and latency minimization, and throughput and spectral efficiency maximization. The total optimization function based on a weighted sum approach is calculated as:

$$
f_{T O}=w_{1} f_{P}+w_{2} f_{T}+w_{3} f_{B E R}+w_{4} f_{D}+w_{5} f_{S E}+w_{6} f_{I}
$$

where $w_{1}$ to $w_{6}$ are the adaptive weights of the corresponding fitness functions. The fitness functions in Eq. (1) are: $f_{P}$ is the power minimization function, $f_{T}$ is the throughput maximization function, $f_{B E R}$ is the BER minimization function, $f_{D}$ is the delay minimization function, $f_{S E}$ is the spectral efficiency maximization function, and $f_{I}$ is the interference minimization function. Each of these fitness functions is detailed next.

1) Minimization of Power Consumption: Battery life and power consumed are essential factors for reducing power consumption. The parameters contribute to the fitness for power minimization are bandwidth $(B)$, modulation index $(m)$, coding rate $\left(R_{c}\right)$, time division duplexing $(T D D)$, symbol rate $\left(R_{s}\right)$, contention window size which impacts the number of re-transmissions $C W$, and transmit power $(P)$.

The fitness function to minimize power consumption for $N_{c}$ subcarriers is calculated as:

$$
f_{P}=\left\{\begin{array}{c}
1-\left[\sum_{i=1}^{N_{c}}\left(P_{\max }-P_{i}\right)+\left(B_{\max }-B_{i}\right)+\right. \\
\left.\quad\left(\log _{2} m_{\max }-\log _{2} m_{i}\right)+\left(R_{s} \max -R_{s} i\right)\right] * \\
\quad\left[\frac{1}{N_{c} *\left(P_{\max }+B_{\max }+\log _{2} m_{\max }+R_{s} \max \right)}\right], \\
\text { if } P_{\min } \leq P_{i} \leq P_{\max } \\
0, \text { if } P_{i}>P_{\max },
\end{array}\right.
$$

The subscript $i$ refers to $i^{\text {th }}$ subcarrier of the multicarrier system, and $P_{\max }$ is the threshold for maximum transmission power.

2) Maximization of Throughput: Throughput is another important performance metric and considered as the main optimization objective in certain applications like multimedia. BER causes degradation of system throughput and should thus be maintained at an acceptable level. Throughput depends on the following parameters: bandwidth, coding rate, modulation index, frame size $(L)$, probability of BER $\left(P_{b e}\right)$, contention window size as it affects the frames transmission rate, congestion control mechanism, number of hops which is inversely proportional to throughput, transmission range and percentage of transmit time.

The fitness function for throughput for $N_{c}$ subcarriers is

$$
f_{T}=\left\{\begin{array}{l}
\sum_{i=1}^{N_{c}} \frac{L_{i}}{L_{i}+O+H} *\left(1-P_{b e} i\right)^{L_{i}+O} * R_{c} i * T D D_{i} \frac{1}{N_{c}}, \\
\quad \text { if } T \geq T^{*} \\
0, \text { if } T<T^{*},
\end{array}\right.
$$

Where, $O$ is physical layer overhead, $H$ is MAC and IP layer overhead, and $T^{*}$ the threshold for minimum throughput requirements.

3) Minimization of Bit Error Rate (BER): BER is used to measure the quality of each link in terms of number of errors each bit encountered. Generally BER depends on several parameters like transmit power, modulation type, modulation index, bandwidth, symbol rate, contention window size as it is proportional $P_{b e}$, number of hops and noise power. The fitness function for minimizing BER is expressed as:

$$
f_{B E R}=\left\{\begin{array}{l}
1-\left[\frac{\log _{10}(0.5)}{\log _{10}\left(P_{b e}\right)}\right], \text { if } B E R_{t} \leq B E R \leq B E R^{*} \\
1, \text { if } B E R<B E R_{t} \\
0, \text { if } B E R_{s}>B E R^{*}
\end{array}\right.
$$

where $B E R$ is the calculated BER for a certain solution, $B E R_{t}$ the target BER and $B E R^{*}$ the threshold of maximum tolerable BER.

4) Minimization of Delay: There are numerous applications sensitive to transmission latency, so the latency becomes an essential metric in any wireless communication. The delay objective is represented by the following parameters: modulation index, and frame size. The fitness function to optimize transmission latency is expressed as:

$$
f_{D}=L_{i} *\left(\frac{\log _{2} m_{\max }}{L_{i} \min * \sum_{i=1}^{N_{c}} \log _{2} m_{i}}\right)
$$

where $L_{i}$ is frame size for $i^{t h}$ subcarrier.

5) Maximization of Spectral Efficiency: Spectral efficiency can be defined as the amount of information that can be transmitted over a given bandwidth. The symbol rate and modulation index are used to determine the total amount of information being transmitted. Spectral efficiency is computed as

$$
S_{e}=\frac{R_{s}}{B}
$$


The fitness function to maximize spectral efficiency is expressed as:

$$
f_{S E}=\left\{\begin{array}{l}
\frac{\sum_{i=1}^{N_{c}} \frac{m_{i} * R_{s} i * B_{\min }}{B_{i} * m_{\max } * R_{s} \max }}{N_{c}} \\
0, \text { if } S_{e}<S E R^{*}
\end{array}, \text { if } S_{e} \geq S E R^{*}\right.
$$

where $S E R^{*}$ the threshold for minimum spectral efficiency imposed by QoS. Parameter with subscript max and min represent the maximum and minimum value of that parameter.

6) Minimization of Interference: Interference is a fundamental problem in a shared spectrum environment like cognitive networks. For example, minimizing it is given the highest priority in making spectrum allocation to secondary users in a licensed band. To achieve high throughput with less error rate, minimization of interference is necessary. Transmission parameters such as transmit power, bandwidth, and time division duplexing are used to determine the approximate amount of spectral interference fitness value. the following fitness function is used to minimize interference:

$$
f_{I}=\left\{\begin{array}{l}
1-\frac{\sum_{i=1}^{N_{c}}\left(\left(P_{i} * B_{i} * T D D_{i}\right)-\left(P_{\min } * B_{\min } * 1\right)\right)}{N_{c} *\left(P_{\max } * B_{\max } * 100\right)}, \\
\text { if } P_{\min } \leq P_{i} \leq P_{\max } \\
0, \text { if otherwise }
\end{array}\right.
$$

where $P_{\min }$ and $P_{\max }$ are the boundaries for the allowed transmission power.

\section{Adaptive Multi-ObJective Optimization (AMOS) MECHANISM}

The optimization function specified in Eq. (1) is not linear since the objective functions cannot be treated independently. Therefore, we must make trade-offs among the optimization goals; this is what makes AMOS best fit for this multiobjective optimization problem. AMOS is an evolutionary optimization scheme for making resource management decisions, inspired by GA. It starts with a random population of solutions and evolves to reach an optimal solution. Each solution is represented by a trial, and consists of a string of binary bits which corresponds to transmission parameters. The parameters are encoded into bit strings trials using binary encoding and quantized a priori, which has power-of- 2 ranges. The trials are evaluated using objective fitness functions to determine the set of best solutions that meet the QoS requirements. The higher the obtained score of this function, the better the solution.

AMOS algorithm for transmission parameters adaptation is explained in Algorithm 1. First, the objective functions contribution and search spaces are specified based on the application type. For example, if the application was multimedia, then the weight of the throughput fitness function will be the maximum. However, all the other five objective functions will participate in the optimization process but with less weight. Then, AMOS extracts radio environment parameters from the cross-layer interfaces of the designed cognitive architecture. The weights of the fitness functions are updated in real-time according to the current channel conditions. For example, if the channel quality degrades, the weight of BER increase and the weight of throughput is reduced to compensate for the

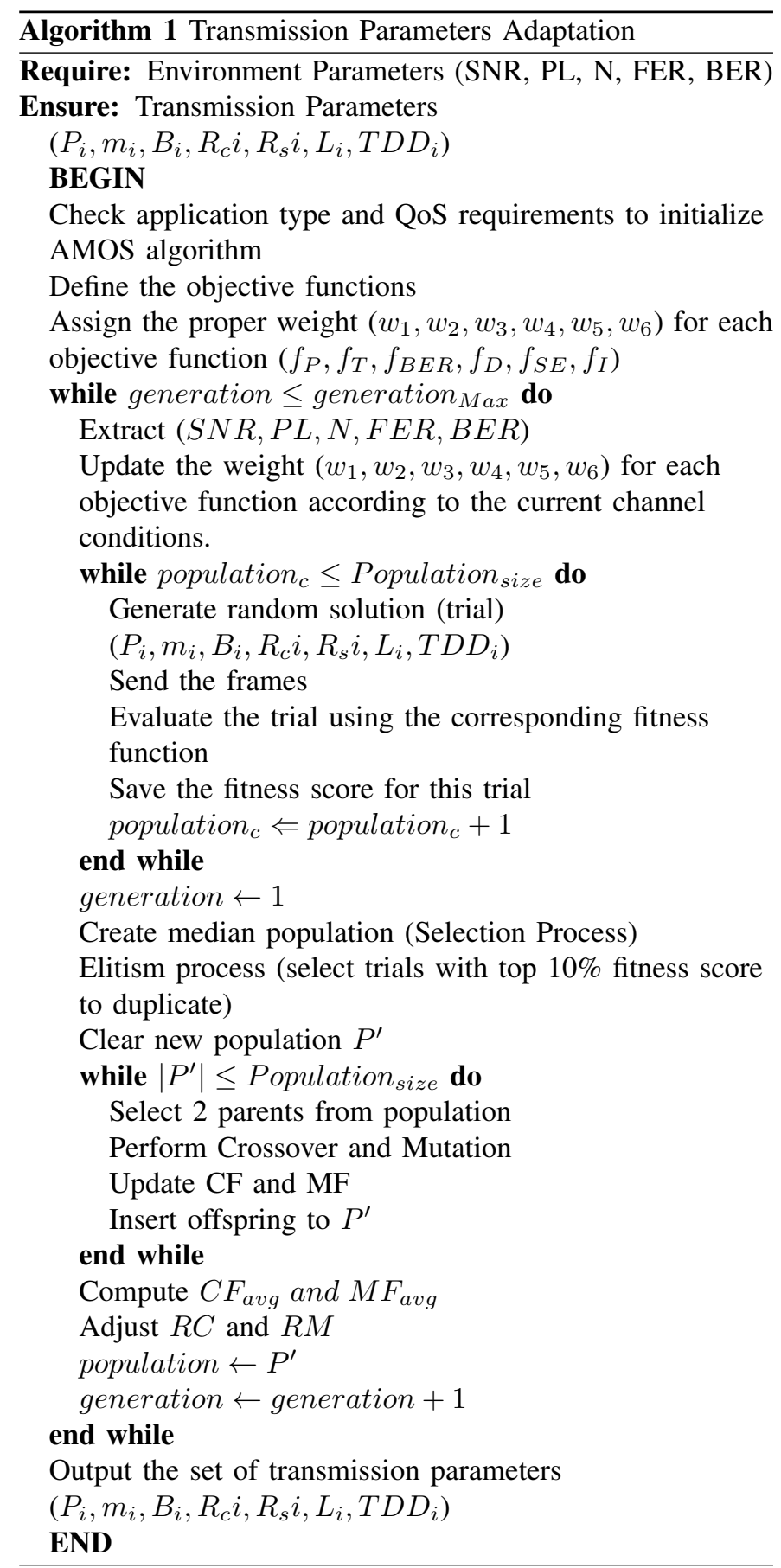

poor channel. The process of weights selection and update in real-time is the responsibility of the reasoning module of the cognitive architecture [14]. Next, an initial random trial $x=P_{1}, P_{2} \ldots P_{N}, m_{1} \ldots m_{N}, B 1, \ldots B_{N}$ is generated and used to transmit the first frame. Once the frame is successfully received, the trial is evaluated using Eq. (1) and the transmission parameters are adjusted. Hence, a new trial is generated to transmit the next frame. The evaluation process is repeated again to generate a new better solution until the maximum number of desired solutions in the population is 
reached (Populationsize). Then, the selection process starts to create median population. Reminder Stochastic Sampling is exploited as a selection method according to [15]. During the selection process, the population is assumed to form a pie chart where each solution trial is assigned a space in the chart that is proportion to its fitness value. Next, an outer roulette wheel is placed around the pie with (Populationsize) equallyspaced pointers. A single spin of the roulette wheel will now simultaneously pick all the members of a median population. After the selection is complete, the median population is created. An Elitism of $10 \%$ is considered to duplicate the best fit set of solutions to the next population to make the algorithm converges faster. Elitism is defined as the process of selecting the best trials from the given solutions set according to the total fitness measure and adding them to the new solutions set without performing crossover and mutation. However, the elitism percentage is maintained to be low to reduce the penalty of limiting the opportunities of exploring other solutions.

Crossover and mutation operations are then applied to the remaining trials. In the crossover process, two trials are recombined in a single point with each other to form two new offsprings [16]. Mutation is an operation of altering binary bit from zero to one, or vice verse, applied to trials after crossover [17]. The choice of the crossover and mutation rate is known to critically affect the behavior and performance of the adaptation scheme. The crossover rate controls the capability of exploiting trials to reach the local optima. The higher the crossover rate, the quicker exploitation proceeds. A large crossover rate would disrupt solutions faster than they could be exploited. The mutation rate controls the speed of exploring a new set of solutions. Mutation rate is always chosen to be small avoid damaging the structure of the trial. Adaptive crossover and mutation rates can, however, traverse different search directions in the state space, thus affecting the performance of the applied algorithm. The overall performance of our algorithm depends on it maintaining an acceptable level of productivity through-out the process of evolution. Thus, it is essential to choose the appropriate crossover and mutation rates. We have employed an adaptive crossover and mutation techniques that adjust crossover and mutation probability dynamically. The first step in our approach is determining an initial crossover and mutation rate $(R C)$ and $(R M)$. We choose a large initial $R M$ to help to explore more solutions and locate a prospective area quickly. During this time, crossover should occur with a small probability to retain diversity. Thus, $R C$ and $R M$ are selected to be 0.6 and 0.4 respectively. Then, crossover and mutation factors are defined as $C F$ and $M F$ as as in Eq. (9) and Eq. (10) respectively.

$$
\begin{gathered}
C F=f_{\text {parents }}-f_{\text {offsprings }} \\
M F=f_{\text {new }}-f_{\text {old }}
\end{gathered}
$$

where $f_{\text {parents }}$ is the sum of fitness function of the parents and $f_{\text {offsprings }}$ is the sum of fitness functions of offsprings. The fitness function of the offesprings after and before mutation are denoted by $f_{\text {new }}$ and $f_{\text {old }}$ respectively. At the end of every crossover and mutation process, $R C$ and $R M$ are adjusted according to Eq. (11) and Eq. (12) respectively.

$$
R C=\left\{\begin{array}{l}
R C+\eta 1, \text { if } C F_{\text {avg }}>M F_{\text {avg }} \\
R C-\eta 1, \text { if } C F_{\text {avg }}<M F_{\text {avg }}
\end{array}\right.
$$

and

$$
R M=\left\{\begin{array}{l}
R M+\eta 2, \text { if } M F_{\text {avg }}>C F_{\text {avg }} \\
R M-\eta 2, \text { if } M F_{\text {avg }}<C F_{\text {avg }}
\end{array}\right.
$$

where $C F_{\text {avg }}=\frac{\sum C F}{n_{c}}$ and $M F_{\text {avg }}=\frac{\sum M F}{n_{c}}$ which are the average of $C F$ and $M F$ respectively, and $n_{c}$ is the number of times crossover and mutation processes are executed. The choice of $\eta 1$ and $\eta 2$ is adaptive according to the convergence of the algorithm. $\eta 1$ and $\eta 2$ are calculated according to Eq. (13):

$$
\eta 1=\eta 2=\left\{\begin{array}{l}
0.02 \frac{f_{\max }-f_{a v g}}{f_{\max }-f \min }, \text { if } f_{\text {max }}>f_{\text {min }} \\
0.02, \text { if } f_{\text {max }}=f_{\text {min }}
\end{array}\right.
$$

where $f_{\text {max }}, f_{\text {avg }}, f_{\min }$ are the maximum, average, and minimum fitness value of trials in the population respectively. After crossover and mutation, a new iteration of AMOS will start and the new population will be re-evaluated during the transmission of the next frames and the process will continue until the stopping criteria is met. The execution of AMOS stops when either of the following occurs: convergence to a stable solution or if the maximum number of generations is reached.

One thing to note is that our scheme tackles the problem of getting stuck in local optima. The adaptive crossover and mutation rates increase the solution space diversity and introduce new possibilities. In addition, the selection method used to create the median population tends to diversify the resulted solutions. All these factors helps the algorithm does not get stuck in local optima. The AMOS implementation also ensures that the final solution is compatible with policy regulations by setting limits in fitness functions for the transmission parameters such as power and frequency which make policy verification easier by the policy layer of the cognitive architecture [14].

\section{EVAluation}

In this section, the performance of AMOS is evaluated using Matlab simulation. we simulate a multicarrier system with $N_{c}=64$ subcarriers with sufficient cyclic prefix is assumed. Each subcarrier is assigned a random attenuation value $\left|H_{i}\right|^{2}, i=1,2 \ldots . N_{c}$ with chi-square distribution. Hence, the signal-to-noise ratio (SNR) varies independently from one subcarrier to another and induces a need for the power and rate adaptation for each individual subcarrier. The channel was assumed to be "block-invariant", implying that the transmission channel impulse response remains constant or undergoes only minor changes over several consecutive frame transmissions. The network topology, is assumed to be uniformly randomly distributed over a given area with consideration of 

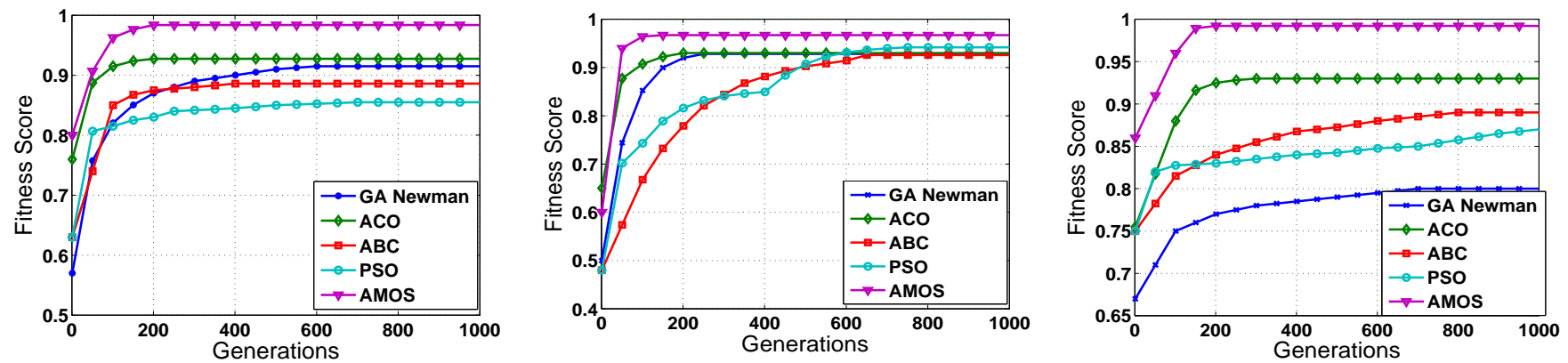

(a) Fitness score comparison in low power scenario (b) Fitness score comparison in multimedia scenario (c) Fitness score comparison in emergency scenario

Fig. 1. Average fitness score comparison in different Scenarios

the Rayleigh-distributed channel coefficients. Other simulation parameters are stated in Table I.

\begin{tabular}{|l||l|}
\hline Parameter & Range \\
\hline Population size & 50 \\
\hline Number of generations & 1000 \\
\hline Congestion control & Depends on the buffer size \\
\hline Number of hops & Multi-hop \\
\hline Transmission range & $\begin{array}{l}\mathrm{d}, \mathrm{d} / 2, \mathrm{~d} / 4 \text { and d/8 where d is the } \\
\text { distance between communicating nodes }\end{array}$ \\
\hline Channel coding rate $\left(R_{c}\right)$ & $1 / 2,1 / 3,2 / 3,3 / 4$ \\
\hline $\begin{array}{l}\text { Contention window size } \\
(C W)\end{array}$ & $\begin{array}{l}\text { CWmin } 8 \text { values between } 4 \text { and } 64 . \\
\text { CWmax } 8 \text { values between } 32 \text { and } 4096\end{array}$ \\
\hline Frame size $(L)$ & 24 bytes to 3072 bytes \\
\hline Bandwidth $(B)$ & 2 to $128 \mathrm{MHz}$ \\
\hline Modulation type and order & M-PSK and M-QAM \\
\hline Transmission power $(P)$ & $0.09 \mathrm{mw}$ to $5 \mathrm{mw}(-24 \mathrm{dBm}$ to 24 dBm) \\
\hline Symbol Rate $(R s)$ & $125 \mathrm{Ksps}$ to $4 \mathrm{Msps}$ \\
\hline $\begin{array}{l}\text { Time Division Duplexing } \\
\text { (TDD) }\end{array}$ & $1 \%$ to $100 \%$ \\
\hline Noise power & $-30 \mathrm{dBm}-5 \mathrm{dBm}$ \\
\hline
\end{tabular}

TABLE I

PARAMETERS USED IN SIMULATION

The simulation targets three different scenarios to demonstrate the performance of AMOS through the fitness score for all subcarriers and the speed of convergence. The first scenario is low power mode scenario where the most contributing objective functions are minimization of power consumption and interference. The second scenario is the multimedia scenario where delay minimization and throughput maximization are the main objectives. The last scenario is the emergency scenario where reliability is the transmission objective and this is represented by minimization of BER. Spectral efficiency is a generic objective that is essential in all transmission scenarios. In this simulation, we compare AMOS with other metaheuristic searching algorithms including Ant-colony algorithm (ACO) [9], Artificial Bee Colony Algorithm (ABC) [18], Particle Swarm Optimization (PSO) [10] and GA proposed by Newman in [19]. All these scheme aims to adapt radio parameters in a multi-carrier environment. Figure 1(a), 1(b), and 1(c) show the fitness score of the five schemes for low power, multimedia, and emergency scenarios respectively. AMOS achieves the highest score and has the fastest convergence compared to the other schemes. All the comparable schemes lacks adaptativty in decision-making as their weights of the objective functions are fixed regardless the continuous variation in the environment conditions. In addition, they focus only on physical layer parameters optimization. Both PSO and $\mathrm{ACO}$ are based on random decision and require more information for parameters selection. Newman GA uses fixed crossover and mutation rate and does not consider essential objectives such as delay and spectral efficiency.

We have conducted another set of simulation to demonstrate AMOS adaptation with respect to TCP/IP stack layers. The first simulation focuses on physical layer parameters optimization. AMOS is compared with the conventional scheme which is based on predetermined transmission parameters in addition to searching schemes compared before. Figure 2(a) presents the achieved throughput as a function of SNR for all the compared schemes with target BER equal $10^{-} 3$. AMOS outperforms other schemes as it achieved the highest throughput through all evaluation scenarios. Low SNR represents the emergency and low power scenario which favors reliability and power consumption on the account of the throughput. High SNR values represent the multimedia scenario which targets throughput maximization on the expense of power consumption and reliability. The conventional standard has the worst performance because it uses the same modulation order over all sub-carriers. The second simulation considered cross layer optimization of AMOS in addition to physical layer optimization. Mainly, it aims to show the effect of adapting MAC layer parameters which include contention window and frame size. The choice of higher minimal contention window size $\left(C W_{\min }\right)$ reduces power consumption and increases the throughput. Figure 2(b) shows a comparison between AMOS, the conventional scheme and "PHY then MAC" optimization scheme. "PHY then MAC" adapts physical- and MAClayer parameters separately. The physical-layer parameters are optimized using the algorithm in [20] while the MAC-layer parameters are optimized using an exhaustive search. AMOS achieves better throughput than both schemes. The reason is that the conventional scheme uses prtedermined transmission parameters and "PHY then MAC" optimizes the contention window size and frame size based on target BER and not the current BER value. This leads to non optimal transmission parameters when the current BER is much smaller than the 


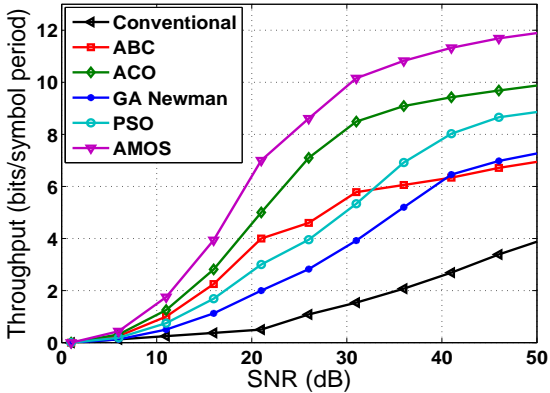

(a) Throughput of different schemes with physical layer optimization

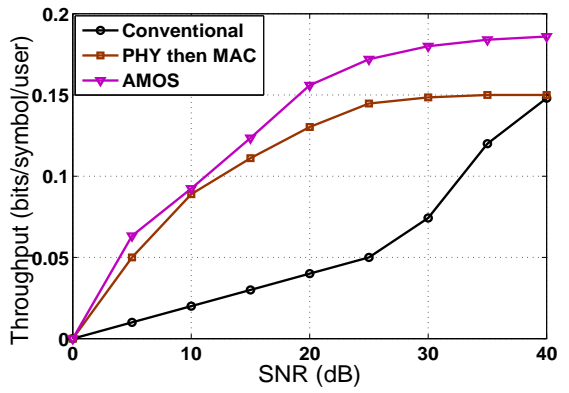

(b) Throughput of differen
MAC) layers optimization

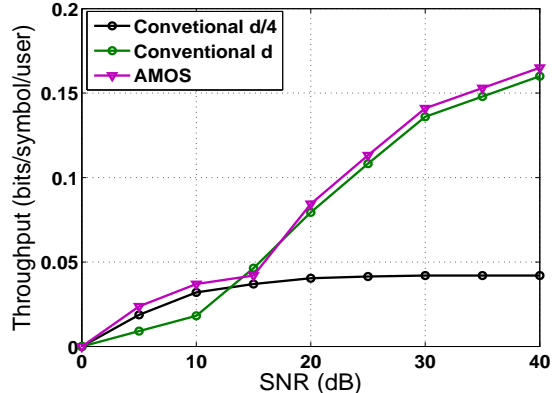

(c) Throughput of different schemes with crosslayer optimization

Fig. 2. Throughput comparison for different optimization schemes as function of SNR

target BER at high SNR values. The third simulation focuses on network layer parameters adaptation which include transmission range and number of hops as well as physical and MAC layer transmission parameters. In this simulation, we compare AMOS which has the capability to adapt transmission range and number of hops dynamically with conventional scheme that uses fixed transmission range $d / 4, d$ (four hops and one hop respectively). Figure 2(c) shows that at low SNR, it is better to use more hops to ensure reliability. However, fewer hops are the best practice when SNR is high as the channel reliability is enough to transmit for longer distances. AMOS is able to be consistent with the channel condition by modifying the transmission range and changing number of hops.

\section{CONCLUSION}

In this paper, we proposed an adaptive multi-objective optimization scheme for cognitive radio network with multiple sub-carriers. AMOS is employed for the optimization phase in the CR's decision-making engine using six objective functions. We demonstrated how the designed scheme can exploit the collected network state information to improve network performance while meeting the QoS requirements. The simulation results have shown the feasibility of AMOS as an optimization scheme which is the core part of decision-making in cognitive engines. AMOS yields results that outperforms other optimization schemes for multicarriers environment. In future, we would like to conduct more extensive studies with large-scale networks and investigate issues of overall network capacity constraints.

\section{REFERENCES}

[1] J. Mitola III, Cognitive Radio: An Integrated Agent Architecture for Software Radio, PhD thesis, Royal Institute of Technology (KTH), 2000.

[2] A. Luiz Garcia Reis, A.F. Barros, K. Gusso Lenzi, L.G. Pedroso Meloni, and S.E. Barbin, "Introduction to the software-defined radio approach", Latin America Transactions, IEEE (Revista IEEE America Latina), vol. 10, no. 1, pp. $1156-1161$, jan. 2012.

[3] Petri Mähönen, Marina Petrova, Janne Riihijärvi, and Matthias Wellens, "Cognitive Wireless Networks: Your Network Just Became a Teenager", in Proceedings of IEEE INFOCOM 2006, 2006.

[4] C. Rieser, Biologically inspired cognitive radio engine model utilizing distributed genetic algorithms for secure and robust wireless communications and networking, PhD thesis, Virginia Tech, 2004.
[5] B. Johansson, P. Soldati, and M. Johansson, "Mathematical decomposition techniques for distributed cross-layer optimization of data networks", IEEE Journal on Selected Areas in Communications, vol. 24, no. 8, pp. $1535-1547$, aug. 2006.

[6] H. Ishibuchi, "Evolutionary multiobjective design of fuzzy rule-based systems", in IEEE Symposium on Foundations of Computational Intelligence, april 2007, pp. $9-16$.

[7] L.A. Zadeh, "Fuzzy logic = computing with words", IEEE Transactions on Fuzzy Systems, vol. 4, no. 2, pp. 103 -111, may 1996.

[8] Wei-Feng Shi and Shi-Long Xue, "A novel chaotic neural networks and application", in International Conference on Machine Learning and Cybernetics, aug. 2005, vol. 8, pp. $4651-4656$ Vol. 8.

[9] M. Waheed and A. Cai, "Cognitive radio parameter adaptation in multicarrier environment", in Fifth International Conference on Wireless and Mobile Communications,ICWMC., Aug 2009, pp. 391-395.

[10] Zhijin Zhao, Shiyu Xu, Shilian Zheng, and Junna Shang, "Cognitive radio adaptation using particle swarm optimization.", Wireless Coтmunications and Mobile Computing, vol. 9, no. 7, pp. 875-881, 2009.

[11] E. Meshkova, J. Riihijarvi, A. Achtzehn, and P. Mahonen, "Exploring simulated annealing and graphical models for optimization in cognitive wireless networks", in IEEE Global Telecommunications Conference, GLOBECOM, 2009, pp. 1-8.

[12] V. Petridis, S. Kazarlis, and A. Bakirtzis, "Varying fitness functions in genetic algorithm constrained optimization: the cutting stock and unit commitment problems", IEEE Transactions on Systems, Man, and Cybernetics, Part B: Cybernetics, vol. 28, no. 5, pp. 629-640, oct 1998.

[13] Sue Ellen Haupt Randy L. Haupt, Practical Genetic Algorithms, Addison-Wesley Longman Publishing Co., Inc., 3nd edition, 2004.

[14] I. AlQerm, B. Shihada, and K.G. Shin, "Cogwnet: A resource management architecture for cognitive wireless networks", in 22nd International Conference on Computer Communications and Networks (ICCCN), July 2013, pp. 1-7.

[15] Darrell Whitley, "A genetic algorithm tutorial", Statistics and Computing, vol. 4, pp. 65-85, 1994.

[16] Ouzhan Hasanebi and Fuat Erbatur, "Evaluation of crossover techniques in genetic algorithm based optimum structural design", Computers and Structures, vol. 78, no. 13, pp. 435 - 448, 2000.

[17] Reeves C R and J E Rowe, Genetic Algorithms: Principles and Perspectives A Guide to GA Theory, Kluwer Academic Publishers, 2003.

[18] P.M. Pradhan, "Design of cognitive radio engine using artificial bee colony algorithm", in International Conference on Energy, Automation, and Signal (ICEAS), 2011, pp. 1-4.

[19] Tim R. Newman, Brett A. Barker, Alexander M. Wyglinski, Arvin Agah, Joseph B. Evans, and Gary J. Minden, "Cognitive engine implementation for wireless multicarrier transceivers", Wirel. Commun. Mob. Comput., vol. 7, no. 9, pp. 1129-1142, Nov. 2007.

[20] R.F.H. Fischer and J.B. Huber, "A new loading algorithm for discrete multitone transmission", in Global Telecommunications Conference, nov 1996, vol. 1, pp. $724-728$ vol.1. 